\title{
Conformism and cooperation in a local interaction model
}

\author{
Friederike Mengel
}

Published online: 5 October 2008

(C) The Author(s) 2008. This article is published with open access at Springerlink.com

\begin{abstract}
We analyze a local interaction model where agents play a bilateral prisoner's dilemma game with their neighbors. Agents learn about behavior through payoff-biased imitation of their interaction neighbors (and possibly some agents beyond this set). We find that the Eshel et al. (Am Econ Rev $88: 157-179,1998)$ result that polymorphic states are stochastically stable in such a setting is not robust. In particular, whenever agents use information also of some agents beyond their interaction neighbors, the unique stable outcome is one where everyone chooses defection. Introducing a sufficiently strong conformist bias into the imitation process, we find that full cooperation always emerges. Conformism is thus identified as a new mechanism that can stabilize cooperation.
\end{abstract}

Keywords Cooperation · Imitation · Local interaction • Conformism

JEL Classification $\mathrm{C} 72 \cdot \mathrm{C} 73 \cdot \mathrm{D} 85$

I thank Karl Schlag, Jakub Steiner and Fernando Vega Redondo as well as seminar participants at Budapest (EEA 2007), Cornell and Jena (MPI Spring Workshop) for helpful comments.

F. Mengel $(\varangle)$

Department of Economics (AE1), Faculty of Economics and Business, Maastricht University, P.O. Box 616, 6200 MD Maastricht, Netherlands

e-mail: F.Mengel@algec.unimaas.nl 


\section{Introduction}

In this note, we study a well known result by Eshel et al. (1998). They have shown that, if agents play a bilateral prisoner's dilemma with their interaction neighbors in a circle network and learn about actions via imitation, cooperation can survive in what is called a stochastically stable state. In this paper, we explicitly distinguish between the set of agents one interacts with (interaction neighborhood) and the set of agents one possibly imitates (information neighborhood). We do this because we want to allow for the fact that agents can be informed also about some agents beyond their interaction neighborhood (e.g. their friend's friends) and will use this information when imitating an action. ${ }^{1}$ We find that whenever agents are allowed to hold some information beyond their interaction neighbors, the unique stochastically stable state is one where everyone chooses defection. We then introduce a conformist bias into the imitation process relying on the model by Ellison and Fudenberg (1993). We find that, if this conformist bias is strong enough, full cooperation always emerges irrespective of whether agents hold information beyond their interaction neighbors. Conformism is thus identified as an important and new mechanism that can stabilize cooperation in a local interaction environment.

We also show that the result from Eshel et al. (1998) does not extend to general networks irrespective of whether agents hold information about others beyond their interaction neighbors. In particular, we give examples of asymmetric networks (where not all agents have the same number of neighbors) for which the unique stochastically stable state under payoff-biased imitation involves full defection. Conformism—on the other hand-stabilizes cooperation in these networks.

Previous literature has explained cooperation in networks also through other mechanisms. Marsili et al. (2005) highlight the importance of the clustering degree for sustaining cooperation. Zimmermann et al. (2004), Fosco and Mengel (2008) or Hanaki et al. (2007) among many others explain cooperation through exclusion of non-cooperators in a dynamic network setting. The role of a conformist bias in imitation has been examined by Ellison and Fudenberg (1993) to study the spread of an efficient technology in a one person decision problem. Levine and Pesendorfer (2007) explain cooperation through an imitation process in a set up where agents get some information about the opponent's strategy prior to interaction.

The paper is organized as follows. In Section 2, the model is presented, and in Section 3, the model is analyzed. Section 4 concludes. The proofs are relegated to an Appendix.

\footnotetext{
${ }^{1}$ Alos-Ferrer and Weidenholzer (2006) use differing interaction and information neighborhoods to explain the emergence of the efficient convention in coordination games. See also Galeotti et al. (2006).
} 


\section{The model}

\subsection{The local interaction game}

There are $i=1, \ldots n$ agents interacting in a $2 \times 2$ prisoner's dilemma game through a circle network. Interactions are not necessarily restricted to an agents first order neighbors. For any number $h \in \mathbb{N}_{+}$we denote $N_{i}^{h}$ the set of agents within a radius $h$ of "geodesic" distance to agent $i$. Denote $N_{i}^{Z}$ the set of agents with whom agent $i$ interacts or the "interaction neighborhood" of player $i$. Furthermore the set of agents with whom $i$ interacts $\left(N_{i}^{Z}\right)$ will in general not equal the set of agents about whom $i$ has information. Denote the latter set - the information neighborhood of agent $i$-by $N_{i}^{I}{ }^{2}$ We will assume $I \geq Z$. Let it be a convention that $N_{i}^{Z}$ does not contain the player $i$ herself while $N_{i}^{I}$ does. As an illustration consider the circle with interaction radius $Z=1$ and information radius $I=2$ depicted below.

$$
\ldots \overbrace{(i-2)-\underbrace{(i-1)}_{N_{i}^{Z}}-i-\underbrace{N_{i}^{I}}_{N_{i}^{Z}}-1+1)}^{(i+2)}-(i+3) \ldots
$$

Individuals play a $2 \times 2$ prisoner's dilemma with their interaction neighbors $N_{i}^{Z}$. The set of actions is given by $A=\{C, D\}$ for all players. For each pair of actions $a_{i}, a_{j} \in A$ the payoff $\pi_{i}\left(a_{i}, a_{j}\right)$ that player $i$ earns when playing action $a_{i}$ against an opponent who plays $a_{j}$ is given by the following matrix,

\begin{tabular}{|l|l|l|}
\hline$a_{i} \backslash a_{j}$ & $C$ & $D$ \\
\hline$C$ & $\alpha$ & $\beta$ \\
\hline$D$ & $\gamma$ & $\delta$ \\
\hline
\end{tabular}

We are interested in the case $\gamma>\alpha>\delta>\beta>0$ i.e. the case where matrix (1) represents a Prisoner's dilemma. Assume also that $\alpha>\frac{\beta+\gamma}{2}$, i.e. that cooperation $(C)$ is efficient. ${ }^{3}$ The payoffs at time $t$ for player $i$ from playing action $a_{i}$ are given by ${ }^{4}$

$$
\Pi_{i}^{t}\left(a_{i}^{t}, a_{j}^{t}\right)=\sum_{j \in N_{i}^{Z}} \pi_{i}\left(a_{i}^{t}, a_{j}^{t}\right) .
$$

Denote $\Pi^{t}\left(N_{i}^{I}, a\right)=\frac{\sum_{k \in N_{i}^{I} \mid a_{k}^{t}=a} \Pi_{k}^{t}(\cdot)}{\operatorname{card}\left\{k \in N_{i}^{I} \mid a_{k}^{t}=a\right\}}$ the average payoff of all agents in $N_{i}^{I}$ that choose action $a$ and let $\Pi^{t}\left(N_{i}^{I}, a\right)=0$ if $\operatorname{card}\left\{k \in N_{i}^{I} \mid a_{k}^{t}=a\right\}=0$.

\footnotetext{
${ }^{2}$ When we say that $i$ has information about $j$ we mean that $i$ is informed about $j$ 's action choice and payoff.

${ }^{3}$ All the results go through without this assumption. It sometimes reduces the number of subcases though, that have to be considered.

${ }^{4}$ In Eq. 2 agents get the same payoff from all their interaction partners. One could easily generalize to a situation where — as in the connections model from Jackson and Wolinsky (1996) - payoffs are discounted in proportion to the geodesic distance between the two interaction partners.
} 


\subsection{Learning}

At each point in time $t=1,2,3 \ldots$ the state of the system is given by the action choices of all agents $s(t)=\left(a_{i}^{t}\right)_{i=1}^{n} 4$. Denote $S$ the state space. At each point in time a (small) number $r$ of agents is randomly selected to revise their action choices. We consider two possible decision rules. First we consider the rule typically used in the literature where agents rely on payoff-biased imitation. Then we add a conformist-bias into the imitation process.

\subsubsection{Payoff-biased imitation}

Under the basic process an agent (who is selected to revise her action choice) compares the average payoff in her information neighborhood of the action she is currently not choosing $\neg a_{i}$ and her action $a_{i}$. If and only if

$$
\Pi^{t-1}\left(N_{i}^{I}, \neg a_{i}\right)-\Pi^{t-1}\left(N_{i}^{I}, a_{i}\right)>0
$$

she changes her action. With small probability $\varepsilon$ she trembles and reverses her choice. This is the rule used, for example, by Eshel et al. (1998).

\subsubsection{Payoff- and conformist-biased imitation}

The process with conformism takes into account the possibility that agents might be more inclined to make more "popular" choices. Decision rule (3) is substituted by the following rule,

$$
\Pi^{t-1}\left(N_{i}^{I}, \neg a_{i}\right)-\Pi^{t-1}\left(N_{i}^{I}, a_{i}\right)>m\left(1-2 x_{\neg a_{i}}\right) .
$$

$m \in \mathbb{R}^{+}$is a finite conformity parameter and $x_{\neg a_{i}}$ the share of all agents that $i$ knows about that use a different action then herself, i.e. $x_{\neg a_{i}}=(2 I+$ $1)^{-1} \operatorname{card}\left\{j \in N_{i}^{I} \mid a_{j} \neq a_{i}\right\}$. Obviously $m=0$ corresponds to the basic process. If both actions are equally popular, i.e. if $x_{\neg a_{i}}=1 / 2$, the agent is not biased towards using either of them. If one of the actions is more popular, on the other hand, the agent will be ceteris paribus more inclined to use that action. Decision-rule (4) is essentially the rule used in Ellison and Fudenberg (1993). ${ }^{5}$

\subsection{Techniques used in the analysis}

The learning process described in Section 2.2 (under either decision rule) gives rise to a finite Markov chain, for which the standard techniques apply. Denote $P^{\varepsilon}\left(s, s^{\prime}\right)$ the transition probability for a transition from state $s$ to $s^{\prime}$ and $P^{0}\left(s, s^{\prime}\right)$ the transition probability if $\varepsilon=0$. An absorbing set under $P^{0}$ is a minimal subset of states which, once entered, is never left. An absorbing state is a singleton absorbing set, or in other words,

\footnotetext{
${ }^{5}$ They analyze the adoption of efficient technologies in a one-person decision problem where agents may be subject to a conformist bias.
} 
Definition 1 State $s$ is absorbing $\Leftrightarrow P^{0}(s, s)=1$.

As trembles make transitions between any two states possible, the perturbed Markov process has a unique stationary distribution, denoted $\mu^{\varepsilon}{ }^{6}$ The limit invariant distribution $\mu^{*}=\lim _{\varepsilon \rightarrow 0} \mu^{\varepsilon}$ exists and its support $\{s \in$ $\left.S \mid \lim _{\varepsilon \rightarrow 0} \mu^{\varepsilon}(s)>0\right\}$ is a union of some absorbing sets of the unperturbed process. The limit invariant distribution singles out a stable prediction of the unperturbed dynamics $(\varepsilon=0)$ in the sense that for any $\varepsilon>0$ small enough the play approximates that described by $\mu^{*}$ in the long run. The states in the support of $\mu^{*}$ are called stochastically stable states. These are the states we focus on.

Definition 2 State $s$ is stochastically stable $\Leftrightarrow \mu^{*}(s)>0$.

Denote $\omega$ the union of one or more absorbing sets and $\Omega$ the set of all absorbing sets. Define $X\left(\omega, \omega^{\prime}\right)$ as the minimal number of trembles necessary to reach $\omega^{\prime}$ from $\omega .^{7}$ The stochastic potential $\psi(s)$ of a state $s \in \Omega$ is defined as the sum of minimal trembles necessary to induce a (possibly indirect) transition to $s$ from any alternative state $s^{\prime} \in \Omega$, i.e. $\psi(s)=\sum_{s^{\prime} \in \Omega} X\left(s^{\prime}, s\right)$.

Result (Young 1993) State $s^{*}$ is stochastically stable if it has minimal stochastic potential, i.e. if $s^{*} \in \arg \min _{s \in \Omega} \psi(s)$.

The intuition behind Young's result is simple. In the long run, the process will spend most of the time in one of its absorbing states. The stochastic potential of any state $s$ is a measure of how easy it is to jump from the basin of attraction of other absorbing states to that of $s$ by perturbing the process a little.

\section{Analysis}

Throughout the analysis, we assume that $I$ is small relative to the number of players $n$. In particular, we will assume that $I<\frac{n-2}{4}$, ensuring that, for any agent, at least one other agent can be found such that their information neighborhoods are disjoint. ${ }^{8}$ Let us briefly characterize absorbing states.

Absorbing states States where $a_{i}=a, \forall i \in G$ are absorbing. Furthermore there exists $\bar{\alpha}(m, Z, I)>0$ such that a set of polymorphic states is absorbing whenever

\footnotetext{
${ }^{6}$ See Karlin and Taylor $(1975)$ for a textbook on stochastic processes or Young $(1993,1998)$ for applications of these techniques to economics.

${ }^{7}$ It is important to note that these transitions need not be direct (i.e. they can pass through another absorbing set).

${ }^{8} \mathrm{We}$ want to focus on both local interaction and local information. Without local interaction of course network analysis is pointless.
} 
$\alpha>\bar{\alpha}(\cdot)$. In all such states, there are strings of cooperators separated by strings of defectors.

Proof Appendix.

The exact composition of the set of polymorphic absorbing states depends on the coefficient for conformism $m$ as well as the information radius $I$ and the interaction radius $Z$. In the following, we will denote $s^{a}$ the state where all agents play action $a \in\{C, D\}$ and $\omega^{C D}$ the set of polymorphic absorbing states.

\subsection{Payoff-biased imitation in the circle network}

Start with a situation where $I>Z$, i.e. where agents hold some information about others beyond their interaction neighborhood. Agents can obtain such information for example if their friends tell them about their friends etc. We will show that if agents rely on payoff-biased imitation only the unique outcome in these situations is full defection. As an illustration consider the network depicted in Fig. 1 where $I=2$ and $Z=1$. Then from the fully cooperative state $s^{C}$ one tremble by any player can induce a transition to state $s^{D}$ with full defection. To see this assume that starting from $s^{C}$ player 2 trembles and switches to action $D$. Now player 4 will want to imitate player 2 as the average defector payoff in his information neighborhood $\Pi^{t}\left(N_{4}^{I}(D)\right)=2 \gamma$ exceeds the average cooperator payoff $\Pi^{t}\left(N_{4}^{I}(C)\right.$ ) (Fig. 1).

Consider next player 6 and note that $N_{6}^{I}=\{4,5,6,7,8\}$. Consequently $\Pi^{t}\left(N_{6}^{I}(D)\right)=2 \gamma>\Pi^{t}\left(N_{6}^{I}(C)\right)$ and player 6 will switch to defection. If next

Fig. 1 Circle network with $I=2$ and $Z=1$. Player 2 is a defector

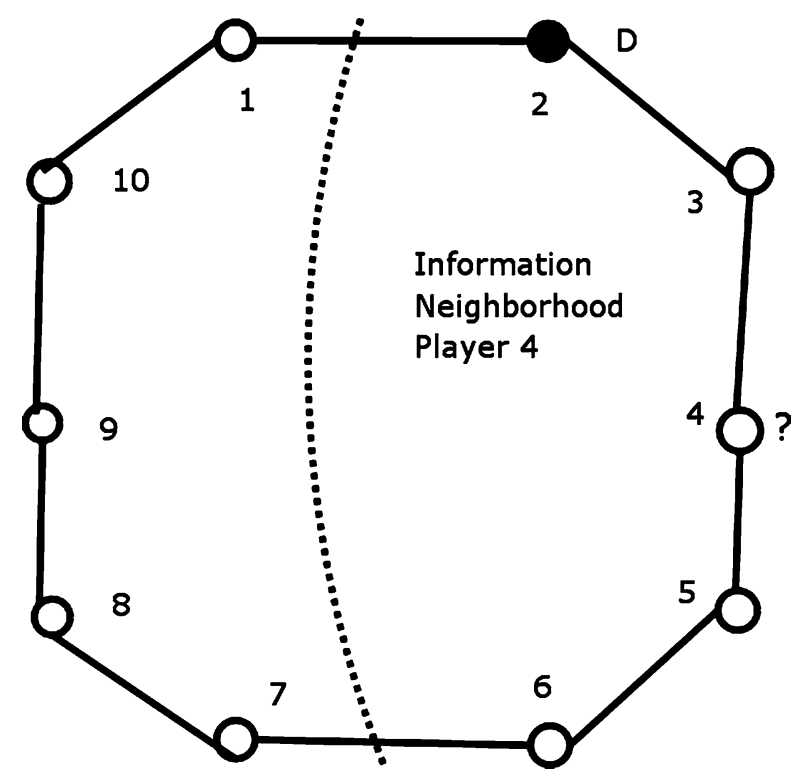


Fig. 2 Circle network with $I=2$ and $Z=1$. Player 6 imitates player 4

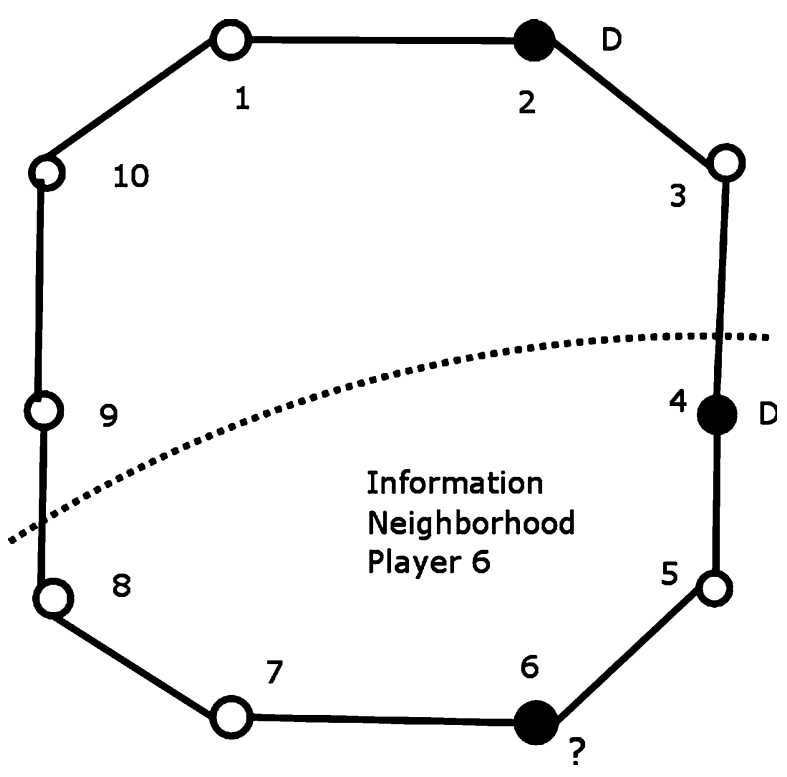

player 8, then player 10,12 etc... switch to defection, then all remaining cooperating players will be surrounded by defectors. Consequently $\Pi^{t}\left(N_{i}^{I}(D)\right)>$ $2 \beta=\Pi^{t}\left(N_{i}^{I}(C)\right), \forall i \in G$ and the remaining cooperators will also want to switch to defection. We end up in $s^{D}$. Such a transition after only one tremble is possible because of the fact that $I>Z$. This allows defection to spread "across long distances", ensuring that cooperators always interact with more defectors than the defectors themselves during the transition. On the other hand, it is clear that, for a transition from $s^{D}$ to a state characterized by some cooperation, more than one tremble is needed as a single cooperator surrounded by defectors will have the minimum possible payoff and will never be imitated. This underlies the fact that the unique stochastically stable state is $s^{D}$ (Fig. 2).

Proposition 1 If $I>Z$ the unique stochastically stable state is $s^{D}$.

\section{Proof Appendix.}

Next we consider the case $I=Z$, previously examined by Eshel et al. (1998). This case reflects situations where agents' information is restricted to their interaction partners. Examples for such situations will be found in anonymous interactions, like e.g. the interaction between buyers and sellers in a supply chain. Now a transition from $s^{C}$ to $s^{D}$ is not always possible after one action tremble. As an illustration consider the network depicted in Fig. 3 where $I=Z=1$. Let player 2 tremble to action $D$. Her action will be imitated by one of her interaction partners as $N_{2}^{I}=\{1,2,3\}=N_{2}^{Z}$ (Fig. 3). 
Fig. 3 Circle network with $I=Z=1$. Player 2 chooses defection

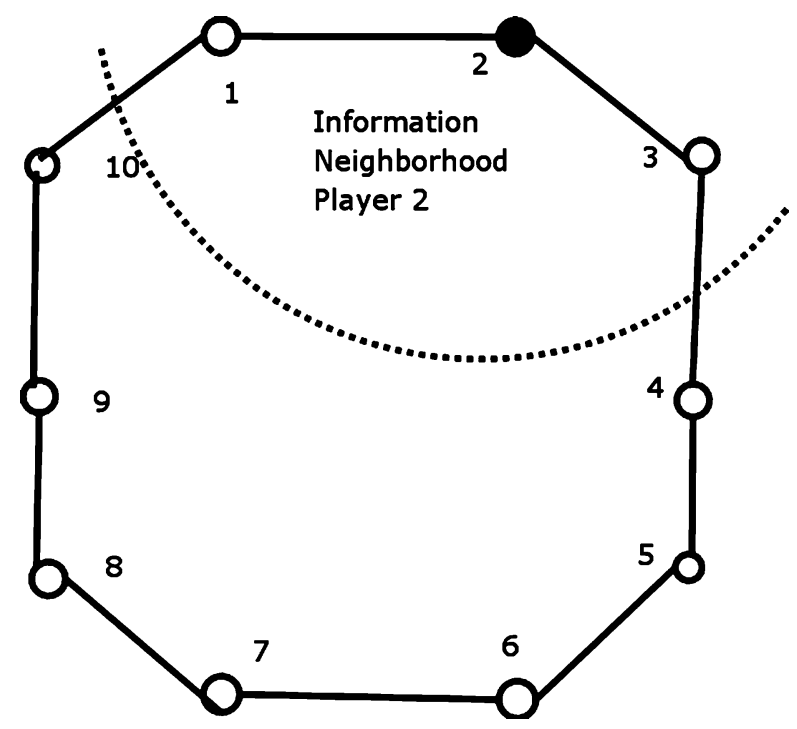

Say player 1 switches to $D$. As $N_{1}^{I} \cup N_{2}^{I}=\{n, 1,2,3\}$, the only players who might adopt the deficient action now are players $n$ and 3 (Fig. 4). The average payoff of cooperating agents in these information neighborhoods is given by $\Pi^{t}\left(N_{n}^{I}(C)\right)=\Pi^{t}\left(N_{3}^{I}(C)\right)=\frac{3 \alpha+\beta}{2}$. The average payoff of defectors is given by $\Pi^{t}\left(N_{n}^{I}(D)\right)=\Pi^{t}\left(N_{3}^{I}(D)\right)=\gamma+\delta$. Defection will spread if and only if $\alpha<\frac{2(\gamma+\delta)-\beta}{3}$. In this case, a transition to $s^{D}$ can be induced via one tremble.

Fig. 4 Circle network with $I=Z=1$. Player 3 imitates player 2

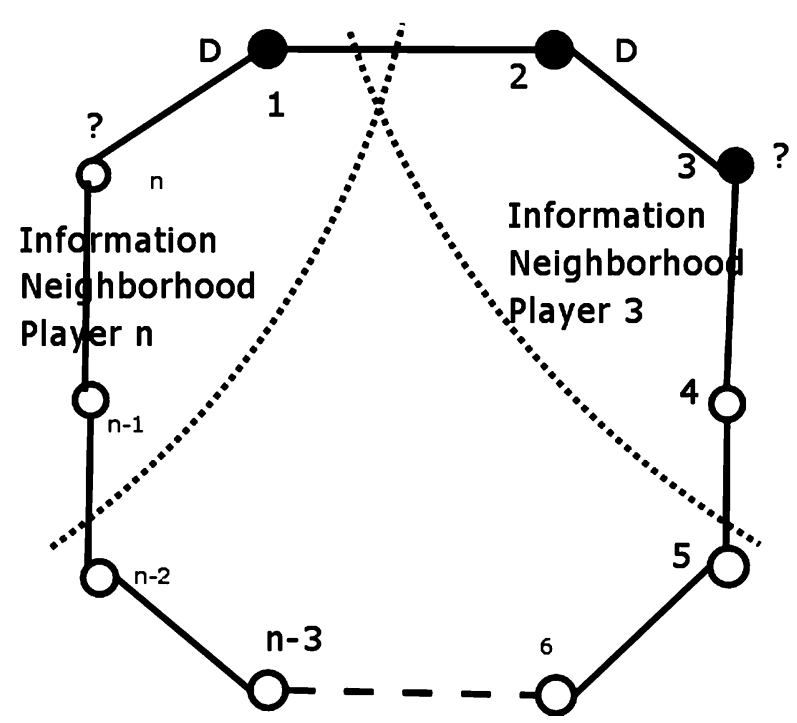


What happens with the reverse transition from $s^{D}$ to $s^{C}$ ? Under some conditions on the payoffs two simultaneous trembles will suffice for the cooperative action to spread through the graph until two defectors are left. These defectors, though, will never want to imitate the cooperative action and we end up in a polymorphic stochastically stable state. More precisely,

Proposition 2 If $I=Z$, there exists $\bar{\alpha}(I, Z)>0$ such that, if the game payoffs satisfy $\alpha \geq \bar{\alpha}(\cdot)$, state $s$ is stochastically stable $\Rightarrow s \in \omega^{C D}$. If $\alpha<\bar{\alpha}(\cdot)$ the unique stochastically stable state is $s^{D}$.

\section{Proof Appendix.}

Less information does actually help cooperation. The reason is that defection now can only spread locally, forcing defectors to interact with each other. This reduces the average payoff of defectors revealing the social benefit of cooperation. Proposition 2 essentially generalizes the result from Eshel et al. (1998). ${ }^{9}$

\subsection{Payoff-biased and conformist-biased imitation in the circle network}

In this section, we assume that agents display a conformist bias, i.e. that they are more inclined to imitate more popular actions. We show that, if imitation is payoff-biased and (sufficiently) conformist-biased the unique stochastically stable state involves full cooperation. Consider Fig. 5 where $I=2$ and $Z=1$. Now starting from $s^{C}$ assume that player 3 trembles and switches to $D$. Player 1 will now imitate player 3 if and only if the payoff advantage of defection is high enough to make up for the "unpopularity" of this action.

It is shown in the Appendix that a necessary condition for action $D$ to spread through the whole graph after just one tremble is $m<\frac{(2 I+1)[2 I Z(\gamma-\alpha)+Z(\alpha-\beta)]}{(2 I-1) I}$. What happens if agents display a larger degree of conformism? Then (at least) one more tremble in agent 1's information neighborhood is needed for him to switch. Assume player 1 is willing to imitate $D$ after both players 3 and $n-1$ have switched to $D$. Can the defection spread beyond $N_{1}^{I}$ ? Consider the decision of player 4 . His information neighborhood is given by $N_{n-2}^{I}=\{2,3,4,5,6\}$. Players 4, 5 and 6 are cooperators. As one defector is not enough to induce imitation, both 2 and 3 have to play defect for defection to spread through the operation of the unperturbed dynamics alone. But then we have a string of interacting defectors ...1-2-3-4... A small amount of conformism can thus be enough to force transitions in which defectors interact mainly among each other. This reduces the payoff advantage of defectors compared to cooperators, revealing the social benefit of cooperation.

\footnotetext{
${ }^{9}$ They consider the cases where $I=Z=1$ and $I=Z=2$. Another difference is that in their model all agents revise their strategy each period (i.e. $r=n$ ). They claim though that their results are robust to a situation in which $r<n$ and indeed we show that this is the case.
} 
Fig. 5 Circle network with conformist bias

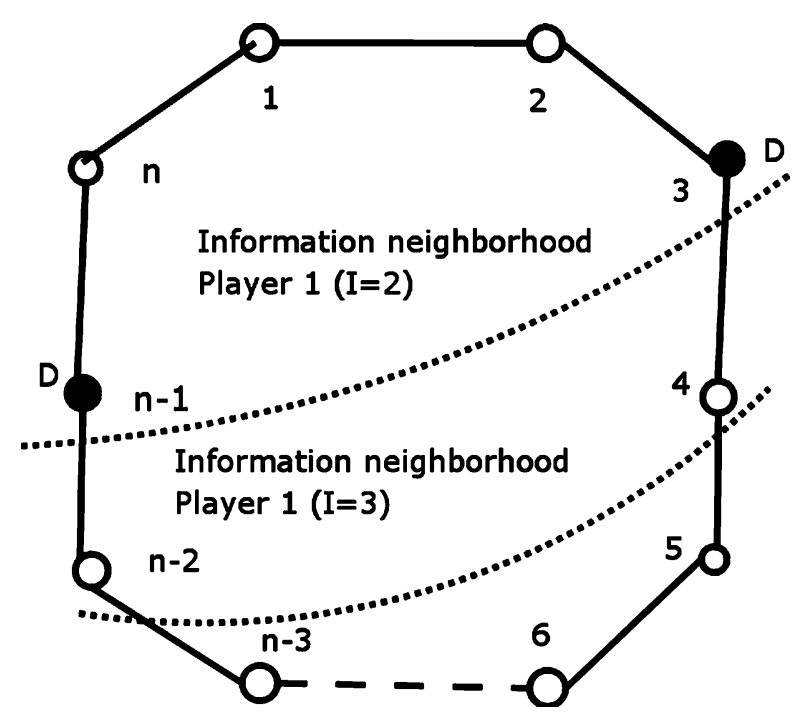

Let us consider now the reverse transition from $s^{D}$ to $s^{C}$. As always, transitions after one tremble are not possible as single cooperators will never be imitated. Depending on the degree of conformism more or less simultaneous trembles are needed in a given information neighborhood to induce a transition. Note though that there is a positive feedback effect, as more trembles of connected cooperators increase the payoff advantage of cooperation over defection. This in turn reduces the need for cooperation to be "popular" in order to spread. Higher degrees of conformism thus favor cooperative outcomes. We can state the following proposition.

Proposition 3 Assume $I>Z$ and that agents display a conformist bias. There exist $\underline{m}(Z, I)>0$ and $\bar{m}(Z, I)>0$ s.th. if $m \geq \bar{m}(\cdot)$ the unique stochastically stable state is $s^{C}$. If $m \leq \underline{m}(\cdot)$ Proposition 2 applies. Furthermore, $\bar{m}(\cdot)$ and $\underline{m}(\cdot)$ are strictly decreasing in $I$ and increasing in $Z$.

Proof Appendix.

Conformism can stabilize cooperation. Furthermore if imitation is conformist-biased more information (larger $I$ ) actually helps cooperation. The intuition is as follows. Conformism is helpful to sustain cooperation because it requires the formation of strings of cooperators or defectors during any transition. But then-given that these strings exist-more information is helpful to achieve cooperation because it enables agents to "look deeper" into the strings. This increases the number of cooperators interacting with cooperators and of defectors interacting with defectors in any agents sample and makes more evident the higher payoff that cooperation yields to a community. 
The case $I=Z$ confirms the results from the case where $I>Z$. Again conformism is helpful to sustain cooperation as the following proposition shows.

Proposition 4 Assume $I=Z$ and that agents display a conformist bias. There exist $m(Z)>0$ and $\bar{m}(Z)>0$ s.th. if $m \geq \bar{m}(\cdot)$ the unique stochastically stable state is $s^{C}$. If $m \leq \underline{m}(\cdot)$ Proposition 3 applies.

Proof Appendix.

\subsection{Other networks}

In this section, we would like to point to another dimension in which the Eshel et al. (1998) result is not robust, but where imitation with a conformist bias yields cooperative outcomes in the long run. In particular we want to discuss two asymmetric networks (where not all players have the same number of nodes) and show that, while with decision rule (3) stochastically stable outcomes yield defection, cooperation is obtained with a conformist bias.

Consider first the interconnected star network depicted in Fig. 6. ${ }^{10}$

In this network there are two types of agents-some "centers" with a "high" degree $k$ (like agent 1 and 2) and some "spokes" with degree one. The stars are interlinked as the centers are linked to $k^{\prime}<<k$ other centers. Assume $I=Z$ as in the original model from Eshel et al. (1998). A transition from any state to $s^{D}$ can occur via one tremble by one of the centers, infecting first all the centers and then the "spokes". As the reverse transitions always need more than one tremble, with decision rule (3) the unique stochastically stable state will be $s^{D}$. What happens under decision rule (4)? If the conformist bias is strong enough agents in the periphery will always conform to what the center does (their unique neighbor). A single action tremble by the center can then infect any star. Now if $I>Z$, a small number (how small depending on $m, I, Z$ ) of infected stars suffices to induce a transition to $s^{C}$, as agents in the cooperative stars will earn higher profits than agents in defective stars. For transitions to $s^{D}$, though, all centers have to tremble simultaneously if the conformist bias is strong enough.

Proposition 5a If $I \geq Z$, the unique stochastically stable state in the interconnected star is given by $s^{D}$ under decision rule (3). There exists $\bar{m}(Z, I)$ s.t. whenever $m>\bar{m}(\cdot)$ the unique stable state is $s^{C}$, if $I>Z$. If $I=Z$ both states $s^{C}$ and $s^{D}$ are stable.

\footnotetext{
${ }^{10} \mathrm{Goyal}$ (2007) points out that defection is the only outcome in a single star if $I=Z$ under decision rule (3).
} 
Fig. 6 Interconnected star network

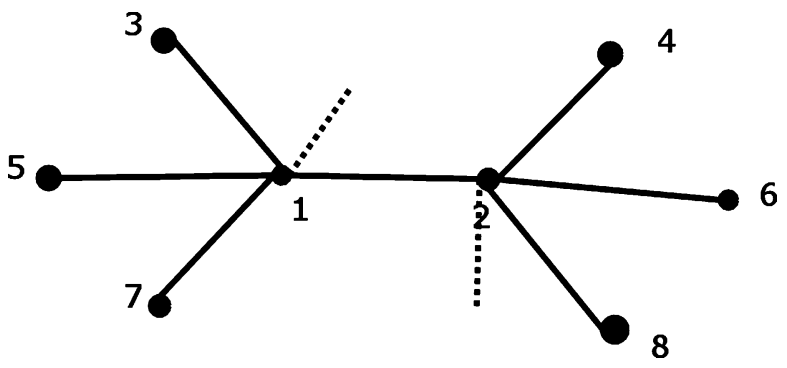

Proof Appendix.

Of course the interconnected star is a network with extreme asymmetries in degree and maybe cooperation obtains even under Eq. 3 as long as the asymmetry is not too extreme. Consider thus the crystal network depicted in Fig. 7. In this network there is an agent $i$ with degree $d$ (in Fig. $7 d=6$ ) whose first-order neighbors have degree $d-1$, whose second-order neighbors have degree $d-2$ and so on until some minimal degree. Again for this network the unique stochastically stable state (with $I=Z$ ) is one where everyone chooses defection. Defection can spread after a tremble by player $i$, infecting one player $j \in N_{i}^{1}$, then one player $k \in N_{j}^{1} \cap \backslash N_{i}^{1}$ and so on. ${ }^{11}$ Again decision rule (4) in this example leads to cooperation (whenever $m$ is "large enough"), because conformism forces actions to spread locally thereby revealing the benefit of cooperation.

Proposition 5b Assume $I \geq Z$. The unique stochastically stable state in the crystal network $(C)$ is given by $s^{D}$ under decision rule (3) and by $s^{C}$ under rule (4), whenever $m>\bar{m}(I, Z)$ for some finite $\bar{m}(I, Z)>0$.

Proof Appendix.

\section{Conclusions}

We have studied a model where agents interact in a prisoner's dilemma through a local interaction structure. Agents learn about optimal actions through imitation. The set of agents they possibly imitate (their information neighborhood) can differ from the set of agents they interact with (their interaction neighborhood). If agents rely on payoff biased imitation alone,

\footnotetext{
${ }^{11}$ Note that this continues to be true if one uses per-link payoffs instead of total payoffs of each agent to calculate $\Pi^{t}\left(N_{i}^{I}(a)\right)$. 
Fig. 7 Crystal network

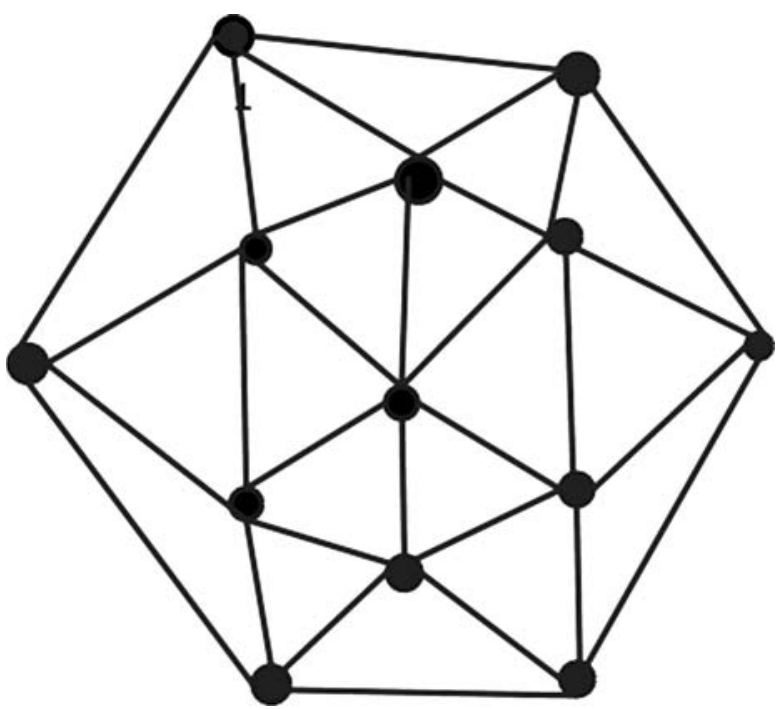

choosing the action (cooperation or defection) that has yielded the higher payoff in the previous period, we find the following results.

- If the information radius of agents exceeds their interaction radius the unique stochastically stable outcome is full defection. Only if information radius and interaction radius are the same can some cooperation be obtained in a stochastically stable state. In this sense more information hurts cooperation.

We then introduce a conformist bias into imitation, assuming that agents are more likely to adopt more "popular" actions and find the following.

- If the conformist bias is large enough all stochastically stable outcomes involve cooperation.

- If there is a conformist bias more information helps cooperation.

The intuition is as follows. Because joint cooperation is beneficial for a community but not individually optimal, strings of cooperators are better off than strings of defectors, whereas single defectors are always better off than single cooperators. But then a larger information radius hurts in the standard case because it allows imitation across "long distances". This works against the formation of strings. Intuitively what conformism does is that it forces actions to spread "locally" thereby revealing the benefit of cooperation. A larger information radius helps in this case because it allows agents to "look deeper" into strings of cooperators and defectors. 


\section{Appendix - Proofs}

$s$-trees

For some of the proofs we will rely on the graph-theoretic techniques developed by Freidlin and Wentzell (1984). ${ }^{12}$ They can be summarized as follows. For any state $s$ an $s$-tree is a directed graph on the set of absorbing states $\Omega$, whose root is $s$ and such that there is a unique directed path joining any other $s^{\prime} \in \Omega$ to $s$. For each arrow $s^{\prime} \rightarrow s^{\prime \prime}$ in any given $s$-tree the "cost" of the arrow is defined as the minimum number of simultaneous trembles necessary to reach $s^{\prime \prime}$ from $s^{\prime}$. The cost of the tree is obtained by adding up the costs of all its arrows and the stochastic potential of a state $s$ is defined as the minimum cost across all $s$-trees.

\section{Notation}

Denote by $\omega_{\rho}^{C D}$ the set of polymorphic absorbing states with $\rho$ strings of cooperators and defectors (note that in any polymorphic absorbing state there has to be an equal number of strings of cooperators and defectors). Denote by $\bar{c}(s) \in \mathbb{N}(\bar{d}(s) \in \mathbb{N})$ the length of the longest cooperator (defector) string in state $s$ and $\underline{\gamma}(s) \in \mathbb{N}(\underline{\delta}(s) \in \mathbb{N})$ the length of the shortest such string.

\section{Absorbing states}

Proof It is clear that given our assumptions the two monomorphic states are always absorbing. ${ }^{13}$

(i) Consider polymorphic states and the case $m=0$. Irrespective of the payoff parameters, there have to be at least $Z+2$ cooperators in the largest string of any absorbing state, i.e. $\bar{c}(s) \geq Z+2$. If not the inequality $\Pi^{t}\left(N^{I}, D\right)>\Pi^{t}\left(N^{I}, C\right)$ will always hold for the last cooperator (denote $i$ ) in any string. Denote $j$ a defector at the edge of a string. In order for a state to be absorbing $\Pi^{t}\left(N_{i}^{I}, C\right) \geq \Pi^{t}\left(N_{i}^{I}, D\right)$ and $\Pi^{t}\left(N_{j}^{I}, C\right) \leq \Pi^{t}\left(N_{j}^{I}, D\right)$ have to hold for all strings. These inequalities define for any given length of a string two threshold level $\widehat{\alpha}_{1}(m, Z, I)$ and $\widehat{\alpha}_{2}(m, Z, I)$ ensuring that neither the cooperator $i$ nor the defector $j$ have incentives to change their actions. Whenever $\alpha \in\left[\widehat{\alpha}_{1}(\cdot), \widehat{\alpha}_{2}(\cdot)\right]$ such states will be absorbing. If $\alpha<\widehat{\alpha}_{1}(\cdot)$ the polymorphic state cannot be absorbing, as agents at the end of a cooperative string will always want to switch to defection. What happens if $\alpha>\max \left\{\widehat{\alpha}_{1}, \widehat{\alpha}_{2}\right\}$ ? Defectors at the end of a defector-string will switch to cooperation. Whenever there are $Z+1$ defectors left in any given string the payoff for defection will exceed that for cooperation and

\footnotetext{
${ }^{12}$ See also Young $(1993,1998)$.

${ }^{13}$ Note that we have assumed that $\Pi^{t}\left(N_{i}^{I}(a)\right)=0$ if $\operatorname{card}\left\{k \in N_{i}^{I} \mid a_{k}^{t}=a\right\}=0$. The intuition simply is that no actions can be imitated that are not present in the population.
} 
no more defectors will switch. In general $\widehat{\alpha}_{1}(\cdot)$ and $\widehat{\alpha}_{2}(\cdot)$ depend of course on $I$ and $Z$. Whenever $I$ and $Z$ are small enough though (relative to $n$ ) polymorphic absorbing states always exist.

(ii) If $m>0$ all polymorphic states with cooperator and defector strings larger than $I+1$ that were absorbing for $m=0$ are still absorbing. The reason is that for these strings cooperation (defection) is more popular in the information neighborhoods of all cooperators (defectors), i.e. $x_{\neg a_{i}<}$ $\frac{1}{2}, \forall i \in G$. Furthermore as $\partial \widehat{\alpha}_{1}(\cdot) / \partial m<0$ whenever $\underline{\gamma}(s), \underline{\delta}(s) \geq I+1$ additional such states can be absorbing. For states with strings shorter than $I+1$ the threshold value $\widehat{\alpha}(\cdot)$ rises and thus some states that were absorbing under $m=0$ may fail to be so whenever $m>0$.

\section{Proof of Proposition 1}

Proof Transitions from $s^{C}$ to $s^{D}$ can occur via one tremble, i.e. $X\left(s^{C}, s^{D}\right)=1$. To see this assume cooperator $i$ trembles and chooses $D$. Then $\Pi_{i}^{t}(\cdot)=$ $2 Z \gamma$. Consider agents $j \in\left\{N_{i}^{I} \cap \backslash N_{i}^{Z}\right\} .{ }^{14}$ This set is non-empty as $I>Z$. As $\Pi^{t}\left(N_{j}^{I}(D)\right)=2 Z \gamma>\Pi^{t}\left(N_{j}^{I}(C)\right)$, these agents $j$ will want to switch to $D$. Next consider agents $k \in\left\{N_{j}^{I} \cap \backslash N_{j}^{Z} \cap \backslash N_{i}^{Z}\right\}$. Again $\Pi^{t}\left(N_{k}^{I}(D)\right)>\Pi^{t}\left(N_{k}^{I}(C)\right)$ and agents $k$ will switch to $D$. In this way transitions can occur during which $D$-players always interact with less $D$-players than $C$-players do, ensuring that $\forall i \in G$ the inequality $\Pi^{t}\left(N_{i}^{I}(D)\right)>\Pi^{t}\left(N_{i}^{I}(C)\right)$ always holds during the transition. The transition $s^{D} \rightarrow s^{C}$ on the other hand needs at least two simultaneous trembles, i.e. $X\left(s^{D}, s^{C}\right) \geq 2$. But then also $X\left(s, \omega^{C}\right) \geq X\left(s, \omega^{D}\right)$, $\forall s \in \omega_{\rho}^{C D}$ and consequently $\psi\left(s^{C}\right)>\psi\left(s^{D}\right)$.

Next we will show that $\psi\left(s^{D}\right)<\psi(s), \forall s \in \omega^{C D}$. Consider first states $s \in$ $\omega_{1}^{C D}$ and assume that at time $t$ the "median" cooperator in the string $c(s)$ trembles and switches to $D$. If the deficient action then infects always the next node at distance $Z+1$ from the node last infected the whole cooperator string can be infected, i.e. $X\left(s, s^{D}\right)=1$. To see this note first that initially defection can spread as the unique defector observed has maximal payoff $2 Z \gamma$. After some time $\tau$ the edge of the string will be reached (it is of course possible that $\tau=0$ ). Note next that - for $s$ to be absorbing - at time $t-1$ (before the tremble) the inequality $\Pi^{t-1}\left(N_{k}^{I}(D)\right) \geq \Pi^{t-1}\left(N_{k}^{I}(C)\right)$ has to hold for the defector $k$ at the edge of the defector string and the inequality $\Pi^{t-1}\left(N_{h}^{I}(D)\right) \leq$ $\Pi^{t-1}\left(N_{h}^{I}(C)\right)$ for the cooperator $h$ at the edge of the cooperator string. But at time $t+\tau$ we have that $\Pi^{t+\tau}\left(N_{h}^{I}(D)\right)>\Pi^{t-1}\left(N_{k}^{I}(D)\right) \geq \Pi^{t-1}\left(N_{k}^{I}(C)\right)>$ $\Pi^{t+\tau}\left(N_{h}^{I}(C)\right)$. Consequently the cooperator $h$ will want to switch to defection. On the other hand $X\left(s^{D}, s\right)>1$ as a single cooperator will never be imitated. Now starting from a minimal $s$-tree we can simply redirect the arrow $s \rightarrow s^{D}$, as the transitions with least resistance are direct. This yields an $s^{D}$-tree with $\psi\left(s^{D}\right)<\psi(s)$. Of course it follows from the same argument that a transition from a state $s^{\prime} \in \omega_{2}^{C D}$ to some state $s \in \omega_{1}^{C D}$ can occur after one tremble only

${ }^{14} \backslash N_{i}^{Z}$ denotes the complement of the set $N_{i}^{Z}$. 
and we can show that $\forall s^{\prime} \in \omega_{2}^{C D}, \exists s \in \omega_{1}^{C D}$ s.t. $\psi(s) \leq \psi\left(s^{\prime}\right)$ (again redirecting the arrow $\left.s \rightarrow s^{\prime}\right)$. Replicating this argument one can show that $\forall s^{j} \in \omega_{j}^{C D}, j \geq$ $2, \exists s^{j-1} \in \omega_{j-1}^{C D}$ s.t. $\psi\left(s^{j-1}\right) \leq \psi\left(s^{j}\right)$. It then follows that $\psi\left(s^{D}\right)<\psi(s), \forall s \in \omega^{C D}$ and thus that $s^{D}$ is the unique stochastically stable state.

Proof of Proposition 2

Proof

(i) First note that whenever $\alpha \leq \bar{\alpha}:=\min _{s \in S} \widehat{\alpha}_{1}(0, I, Z \mid d(s), c(s))$ the only absorbing states are $s^{D}$ and $s^{\bar{C}}$. Furthermore in this case a transition from $s^{C}$ to $s^{D}$ can occur after one action tremble only. As for the reverse transition one tremble does not suffice, the unique stochastically stable state is $s^{D}$.

(ii) Next consider the case where $\alpha>\bar{\alpha}$, in which polymorphic absorbing states do exist. We will first show that $s^{C}$ is not stochastically stable, in particular we will show that there exists a state $s^{\prime} \in \omega^{C D}$ s.t. $\psi\left(s^{\prime}\right)<$ $\psi\left(s^{C}\right)$. Consider the state $s \in \omega_{1}^{C D}$ that is obtained from $s^{C}$ via one single tremble by an agent to defection, i.e. where $X\left(s^{C}, s\right)=1$. Such a state always exists as a tremble to $D$ will be imitated by at least one other agent (possibly more) and the unperturbed process will converge to a polymorphic absorbing state. Now take a minimal $s^{C}$-tree and add the arrow $s^{C} \rightarrow s$. Then consider the old path from $s$ to $s^{C}$. For some $s^{\prime}$ on this path two trembles will be needed to reach the next state (as two neighboring defectors will never want to switch to cooperation). Cutting the arrow that leads away from this state $s^{\prime}$ yields an $s^{\prime}$-tree with $\psi\left(s^{\prime}\right)<\psi\left(s^{C}\right)$.

Now we will show that $s^{D}$ cannot be stochastically stable. Consider transitions from $s^{C}$ to $s^{D}$. The minimal number of trembles needed for this transition is proportional to $\left[\frac{n+1}{\underline{\gamma}(0, Z, Z)}\right]$ as all cooperator strings of size $\gamma(\cdot)$ have to be broken. On the other hand the number of trembles needed to reach $s^{C}$ from $s^{D}$ does not increase with the number of players $n$. Now take a minimal $s^{D}$-tree and consider the path from $s^{C}$ to $s^{D}$. Starting from $s^{D}$ redirect arrows until at some state $s$ a cost of $X\left(s^{D}, s^{C}\right)$ is saved (compared to the old path). Add the arrow $s^{D} \rightarrow s$. This arrow will have a cost strictly smaller than $X\left(s^{D}, s^{C}\right)$. Consequently we have constructed an $s$-tree with $\psi(s)<\psi\left(s^{D}\right)$ where $s \in \omega^{C D}$.

\section{Proof of Proposition 3}

Proof

(i) Consider first transitions from $s^{C}$ to $s^{D}$. Assume one player in $N_{i}^{I}$ trembles and chooses $D$. Then $\Pi\left(N_{i}^{I}(D)\right)-\Pi\left(N_{i}^{I}(C)\right)=\frac{2 I Z(\gamma-\alpha)+Z(\alpha-\beta)}{I}$. 
Player $i$ will want to imitate action $D$ according to decision rule (4) whenever $m<\frac{(2 I+1)[2 I Z(\gamma-\alpha)+Z(\alpha-\beta)]}{(2 I-1) I}=: \underline{m}$. Clearly $\frac{\partial \underline{m}}{\partial Z}>0$ and

$$
\frac{\partial \underline{m}}{\partial I}=\frac{(4 I(I+1)-1)[(2 I Z(\alpha-\gamma)-Z(\alpha-\beta)]}{I^{2}(2 I-1)^{2}}<0 .
$$

Consequently $\underline{m}$ is strictly decreasing in $I-Z$. Now whenever $m<\underline{m}$ a transition from $s^{C}$ to $s^{D}$ can occur after a single tremble and Proposition 1 applies.

(ii) Consider again transitions from $s^{C}$ to $s^{D}$ and assume that $\kappa_{D}<\frac{I+1}{2}$ players in $N_{i}^{I}$ tremble simultaneously and choose $D$. The following inequalities hold: $\Pi\left(N_{i}^{I}(D)\right) \leq 2 Z \gamma$ and $\Pi\left(N_{i}^{I}(C)\right) \geq \frac{2 Z \kappa_{D} \beta+\left(I+1-2 \kappa_{D}\right) 2 Z \alpha}{I+1-\kappa_{D}}$ with equality if $\kappa_{D}=1$. Consequently $\Pi\left(N_{i}^{I}(D)\right)-\Pi\left(N_{i}^{I}(C)\right) \leq$ $\frac{2 Z\left(I+1-\kappa_{D}\right)(\gamma-\alpha)-2 Z \kappa_{D}(\alpha-\beta)}{I+1-\kappa_{D}}$. A necessary condition for a transition after $\kappa_{D}$ trembles in $N_{i}^{I}$ to be possible is

$$
m<\frac{(2 I+1)\left[2 Z\left(I+1-\kappa_{D}\right)(\gamma-\alpha)-2 Z \kappa_{D}(\alpha-\beta)\right]}{\left(I+1-\kappa_{D}\right)\left(2 I+1-2 \kappa_{D}\right)} .
$$

Denote this threshold $\widehat{m}^{D}\left(\kappa_{D}\right)$. Next consider the reverse transition from $s^{D}$ to $s^{C}$. Assume $\kappa_{C}<\frac{I+1}{2}$ players tremble simultaneously in $N_{i}^{I}$ and choose $C$. Then: $\Pi\left(N_{i}^{I}(C)\right) \leq \frac{2\left[Z\left(\kappa_{C}-2\right)+1\right] \alpha+2(2 Z-1) \beta}{2 Z \kappa_{C}}$ and $\Pi\left(N_{i}^{I}(D)\right) \geq$ $\frac{2(2 Z-1) \gamma+\left[2 Z\left(I+1-\kappa_{C}\right)-2(2 Z-1)\right] \delta}{2 Z\left(I+1-\kappa_{C}\right)}$. Consequently a necessary and sufficient condition is

$$
\left(\begin{array}{c}
\frac{2\left[Z\left(\kappa_{C}-2\right)+1\right] \alpha+2(2 Z-1) \beta}{2 Z K_{C}} \\
-\frac{2(2 Z-1) \gamma+\left[2 Z\left(I+1-\kappa_{C}\right)-2(2 Z-1)\right] \delta}{2 Z\left(I+1-\kappa_{C}\right)}
\end{array}\right)>m\left(1-2 \frac{\kappa_{C}}{2 I+1}\right) .
$$

Denote $\widehat{m}^{C}\left(\kappa_{C}\right)$ the threshold defined by Eq. 6. Note that condition (6) is also sufficient, as increasing the number of interacting cooperators increases the average payoff of cooperation.

(iii) Now substitute $\kappa$ for $\kappa_{D}$ in Eq. 5 and for $\kappa_{C}$ in Eq. 6 and consider the continuous extension (to $\kappa \in \mathbb{R}^{+}$) of the functions $\widehat{m}^{C}(\kappa)$ and $\widehat{m}^{D}(\kappa)$. Both $\widehat{m}^{C}(\kappa)$ and $\widehat{m}^{D}(\kappa)$ are strictly increasing in $\kappa$, as can be easily verified. Furthermore $\widehat{m}^{D}(0)>0, \widehat{m}^{C}(0)<0$ and $\left|\partial \widehat{m}^{C}(\kappa) / \partial \kappa\right|>$ $\left|\partial \widehat{m}^{D}(\kappa) / \partial \kappa\right|>0, \forall \kappa \in \mathbb{R}$. We are interested in the point $\kappa^{*}$ where $\widehat{m}^{C}(\kappa)$ and $\widehat{m}^{D}(\kappa+1)$ intersect, i.e. $\kappa^{*}$ s.th. $\widehat{m}^{C}\left(\kappa^{*}\right)=\widehat{m}^{D}\left(\kappa^{*}+1\right)$. This intersection defines the level of conformism such that always at least one tremble more is required for the transition from $s^{C}$ to $s^{D}$ than vice versa. Of course eventually we have to focus on $\kappa \in \mathbb{N}$. Denote $\bar{m}=\widehat{m}^{D}\left(\left\lceil\kappa^{*}\right\rceil\right)$ where $\left\lceil\kappa^{*}\right\rceil$ denotes the smallest integer larger than $\kappa^{*}$. The condition is then $\widehat{m}\left(\frac{I+1}{2}\right)>m>\bar{m} \cdot{ }^{15}$ Finally if $m>\widehat{m}\left(\frac{I+1}{2}\right)$ it is easy to see that $s^{C}$ is reached easier from any state than is $s^{D}$.

\footnotetext{
${ }^{15}$ In fact one could also define $\kappa^{-}$such that $\widehat{m}^{C}\left(\kappa^{-}\right)=\widehat{m}^{D}\left(\kappa^{-}-1\right)$ and $\underline{m}^{\text {new }}=\widehat{m}^{D}\left(\left\lfloor\kappa^{-}\right\rfloor\right)$. Of course $\kappa^{-}<\kappa^{*}$. Whenever $m<\underline{m}^{\text {new }}$ transitions to $s^{D}$ need strictly less simultaneous mutations than the reverse transitions.
} 
(iv) Now we will show that, whenever $m>\bar{m}$, only $s^{C}$ is stochastically stable. We have already seen that $X\left(s^{D}, s^{C}\right)<X\left(s^{C}, s^{D}\right)$ whenever $m>\bar{m}$. What about polymorphic states? We have seen above that all such states have an equal number of strings of cooperators and defectors. Furthermore cooperator-strings are at least as long as defector-strings. But then, of course, even if additional trembles are needed for all agents in the circle to imitate the mutant fewer such trembles will be needed for a transition from $s$ to $s^{C}$ than from $s$ to $s^{D}$. Consequently $X\left(s, s^{C}\right) \leq X\left(s, s^{D}\right), \forall s \in \omega^{C D}$ will always hold. Furthermore it should be clear that $X\left(s, s^{C}\right) \leq X\left(s^{C}, s\right), \forall s \in \omega^{C D}$. But then if $m>\bar{m}, s^{C}$ has minimal stochastic potential.

(v) Finally we will show that $\bar{m}$ is strictly decreasing in $I$. It is clear that $\operatorname{sign}\left[\frac{\partial \bar{m}}{\partial I}\right]=\operatorname{sign}\left[\frac{\partial \kappa^{*}}{\partial I}\right]$ as $\widehat{m}^{D}(\kappa)$ is increasing in $\kappa$. The latter derivative can be computed using the implicit function theorem as

$$
\frac{\partial \kappa^{*}}{\partial I}=-\frac{\partial\left(\widehat{m}^{C}-\widehat{m}^{D}\right) / \partial I}{\partial\left(\widehat{m}^{C}-\widehat{m}^{D}\right) / \partial \kappa}{ }_{\mid \kappa=\kappa^{*}} .
$$

The denominator of this expression is strictly positive. In addition, we have seen that $\partial \widehat{m}^{D} / \partial I<0$ and we have

$$
\frac{\partial \widehat{m}^{C}}{\partial I}=\frac{(\gamma-\delta)(2 Z-1)}{Z(I+1-\kappa)}>0 .
$$

Thus the numerator of Eq. 7 is strictly positive and $\frac{\partial \bar{m}}{\partial I}<0$.

Proof of Proposition 4

Proof The proof follows from the proof of Proposition 3 by substituting $I=Z$ into the relevant conditions.

Proof of Proposition 5

Proof

(i) Start with decision rule (3). A transition from any state $s$ to $s^{D}$ can occur via one tremble by one of the centers in the interconnected star. In the crystal a single tremble by the most highly connected cooperator $i$, who is then imitated by $j \in N_{i}^{I}$ s.t. $d_{j}=d_{i}-1$ etc... can lead to a transition to $s^{D}$. On the other hand for the reverse transitions at least two trembles are needed. But then starting from any minimal $s$-tree, add the arrow $s \rightarrow s^{D}$ at a cost of one and cut the arrow leaving $s^{D}$. This yields an $s^{D}$-tree with $\psi\left(s^{D}\right)<\psi(s)$.

(ii) Next consider rule (4) and the interconnected star network. First note that the "spokes" will always imitate the center if the conformist bias is high enough. But then if $I \geq 2$ a simultaneous tremble by a small number $\kappa_{C}$ of connected centers to $C$ can infect $\kappa_{C}-1$ stars. As agents in the cooperative stars will have higher payoffs, cooperation can then infect 
the entire network. If $I>Z$ then the initial infection (through trembles) of one star (i.e. $k+1$ ) agents suffices to induce a transition to $s^{C}$. On the other hand any transition to $s^{D}$ is stopped once a few stars are infected. Centers will not imitate (if the conformist bias is high enough) unless the spokes do so first. But then all centers have to be first infected in this case. If $I=Z$ all centers have to be infected first for both types of transitions.

In the crystal network a transition from any $s$ to $s^{C}$ can occur via at most $\kappa_{C}$ trembles, where $\kappa_{C}$ is such that for an agent $i$ with $d_{\min }$ all but one neighbors cooperate and that there is a connected path of defectors from an agent with $d_{\min }$ to the center. Then cooperation can spread by first infecting $i$, then $i$ 's neighbor $j$ with $d_{\min }$, then $k \in N_{j}^{I}$ s.t. $d_{k}=d_{\min }+1$ then $h \in N_{k}^{I}$ s.t. $d_{h}=d_{\min }$ etc...until finally infecting the center of the crystal. On the other hand it is easy to see, that the number of trembles needed for a transition from any $s$ to $s^{D}$ is linearly proportional to $n$ with proportionality factor exceeding one. But then starting from any minimal $s$-tree, adding the arrow $s \rightarrow s^{C}$ and deleting the arrow starting at $s^{C}$ yields an $s^{C}$-tree with $\psi\left(s^{C}\right)<\psi(s)$.

Open Access This article is distributed under the terms of the Creative Commons Attribution Noncommercial License which permits any noncommercial use, distribution, and reproduction in any medium, provided the original author(s) and source are credited.

\section{References}

Alos-Ferrer C, Weidenholzer S (2006) Contagion and efficiency. Working paper, University of Vienna

Ellison G, Fudenberg D (1993) Rules of thumb for social learning. J Polit Econ 101:612-643

Eshel I, Samuelson L, Shaked A (1998) Altruists, egoists and hooligans in a local interaction model. Am Econ Rev 88:157-179

Fosco C, Mengel F (2008) Cooperation through imitation and exclusion in networks. Mimeo, University of Alicante

Freidlin MI, Wentzell AD (1984) Random perturbations of dynamical systems. Springer, New York

Galeotti A, Goyal S, Jackson M, Vega-Redondo F, Yaariv L (2006) Network games. Mimeo, Caltech

Goyal S (2007) Connections: an introduction to the economics of networks. Princeton University Press, Princeton

Hanaki N, Peterhansel A, Dodds P, Watts D (2007) Cooperation in evolving social networks. Manage Sci 53(7):1036-1050

Jackson MO, Wolinsky A (1996) A strategic model of social and economic networks. J Econ Theory 71:44-74

Karlin S, Taylor HM (1975) A first course in stochastic processes. Academic, San Diego

Levine DK, Pesendorfer W (2007) The evolution of cooperation through imitation. Games Econom Behav 58:293-315

Marsili M, Slana F, Vega-Redondo F (2005) Clustering, cooperation and search in social networks. J Eur Econ Assoc 3:628-638

Young P (1993) The evolution of conventions. Econometrica 61:57-84

Young P (1998) Individual strategy and social structure. Princeton University Press, Princeton

Zimmermann MG, Eguiluz VM, San Miguel M (2004) Coevolution of dynamical states and interactions in dynamic networks. Phys Rev 69:065102-1 